\title{
UAV Detection: A STDP trained Deep Convolutional Spiking Neural Network Retina-Neuromorphic Approach *
}

\author{
Paul Kirkland ${ }^{1[0000-0001-5905-6816]}$, Gaetano Di Caterina ${ }^{1}$, John Soraghan ${ }^{1}$, \\ Yiannis Andreopoulos ${ }^{2}$, and George Matich ${ }^{3}$ \\ 1 The University of Strathclyde, Glasgow, United Kingdom \\ ${ }^{2}$ University College London, London, United Kingdom \\ 3 Leonardo, London, United Kingdom
}

\begin{abstract}
The Dynamic Vision Sensor (DVS) has many attributes, such as sub-millisecond response time along with a good low light dynamic range, that allows it to be well suited to the task for UAV Detection. This paper proposes a system that exploits the features of an event camera solely for UAV detection while combining it with a Spiking Neural Network (SNN) trained using the unsupervised approach of Spike Time-Dependent Plasticity (STDP), to create an asynchronous, low power system with low computational overhead. Utilising the unique features of both the sensor and the network, this result in a system that is robust to a wide variety in lighting conditions, has a high temporal resolution, propagates only the minimal amount of information through the network, while training using the equivalent of 43,000 images. The network returns a $91 \%$ detection rate when shown other objects and can detect a UAV with less than $1 \%$ of pixels on the sensor being used for processing.
\end{abstract}

Keywords: CNN $\cdot$ SNN $\cdot$ STDP $\cdot$ UAV.

\section{Introduction}

Consumer UAVs and micro-UAVs are increasingly available at low cost, allowing their use in commercial applications (inspection, filming and deliveries)[14] and social use by the general public to become more frequent [19]. However, as the number of UAVs in circulation increases, so does the concern for misuse and accidents. A prime example in the UK recently was the closure of airports due to UAV flying over this restricted area [1], with near misses recorded in the UK in 2018 as 117, up 10 times from 4 years ago [5]. Nonetheless, a number of other concerns, other than collisions, exist due to the UAVs ability to carry a small payload: these could contain potentially harmful chemical or explosives or could be used to smuggle illegal goods [6].

\footnotetext{
* Supported by Leonardo, Data Collection in collaboration with University College London.
} 
Detection of UAVs is not trivial due to their small form factor, coupled with the expanse of the search space. They also possess a high range of manoeuvrability while being difficult to discriminate against birds at distant ranges. These features make it difficult for typical detection approaches such as visual, infra-red, audio and radar to detect the UAV in a wide range of situations [6].

This paper presents a novel UAV Detection system, utilising the features of both the Dynamic Vision Sensor (DVS) and Spiking Neural Network (SNN). This end-to-end spiking Neuromorphic system possesses the following range of features: asynchronous functionality, low power consumption, low computational throughput, high dynamic range, high temporal resolution and dynamic relationship with scene environment. The results of a pilot study show that this system is ideal for the task of UAV detection, displaying features that are unmatched by any other single sensor systems.

The remainder of the paper is organised as follows. Section 2 provides background on the sensor and the spiking network used and explaining the unsupervised learning mechanism. Section 3 provides details about the experimental set-up, Section 4 shows off the results of the system and Section 5 has the discussion of these results.

\section{Background}

Neuromorphic engineering combines research from both the neuroscience and computational neuroscience fields that is exploited within an Engineering aspect. The proposed system makes use of three such Neuromorphic approaches, the event-based camera, spiking neural network and spike time dependent plasticity. The respective sensor, neuron model and learning mechanism combine with a traditional Deep Convolutional Neural Network (DCNN) architecture, to capitalise on the characteristic unique to each.

\subsection{Dynamic Vision Sensor - Event Based Camera}

The Dynamic Vision Sensor is a biologically-inspired sensor (silicon retina) created to mimic how human eye perceives motion with their retina: as such the sensor asynchronously transmits the logarithmic light intensity difference (events) on a pixel by pixel level. This replaces the fixed frame rate traditional camera images, with a far more compressed and sparse output, resulting in 1 to 3 orders of magnitude increase in output rate (33 ms traditional to $15 \mu$ s Event Based) [4]. This allows the sensor to have a much higher temporal resolution (in essence a 66000 frames per second super slow-motion camera for up to 800 pixels, as compared to real world frames per second closer to 1-2,000) but without the caveat of the extra processing required for the pixels that didn't change. Another feature is the DVS's high dynamic range, rated at $>120 \mathrm{~dB}$ vs the $<60 \mathrm{~dB}$ of traditional cameras $[4,11]$. This allows the event based camera to see in a wide variety of lighting conditions, from quickly changing brightness conditions, 
to low light ones, where traditional cameras would not be able to detect anything. A comparison of images captured from a DSLR and the DVS, showing UAVs flying in a well lit and low light scene, are illustrated in Figure 1. It can be seen that the DVS camera is able to capture the shape of the UAV in a well lit situation Fig.1(b) and (c) and is also able to capture the shape in the low light situation when the outline of the UAV is indistinguishable in Fig.1(e) and (f). The images in Fig.1 (c) and (f) show a typical post processing median filtering of the images to give better sensor noise suppression.

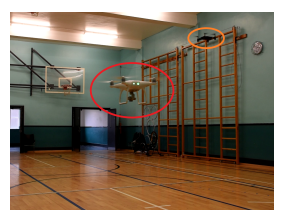

(a)

(Visual Band)

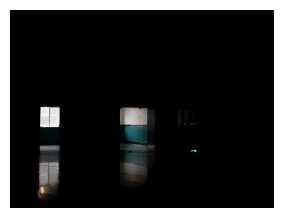

(d)

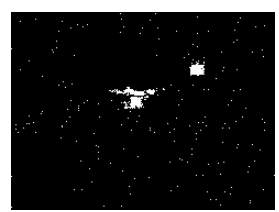

(b)

(Raw DVS Events)

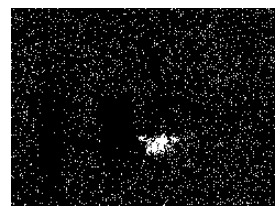

(e)

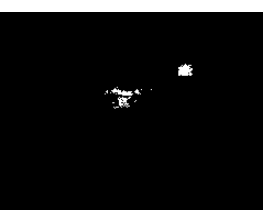

(c)

(Filtered DVS Events)

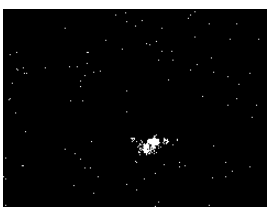

(f)

Fig. 1. Use of the High Dynamic Range within the DVS to capture stark lighting differences. (TOP) Indoor well lit scene (BOTTOM) Low light scene.

The advantages of the DVS leads to the main attribute exploited within this paper, that relates the dynamic relationship to the visual source. This attribute is how the sensor can deliver a sparse yet detailed account of the scene, minimising computation and power. An example of this is shown in Figure 2 highlights the ability to change the integration time of the events captured to create a frame (for visual representation and training). The top row shows a slow-moving UAV, where a higher integration time is required to collect enough event to represent the UAV, as not as many changes in light intensity occur. While the bottom row illustrates the removal of motion blur, in a fast-moving UAV collision, by decreasing the integration time. The integration times can also be overlapped allowing a combination of both a longer integration time to capture events and the fine temporal resolution changes in the scene. The main drawback to the current DVS technology is the low spatial resolution. However, active research in this area has shown cameras with a sensor size of 640x480 [16] and 384x320 [9] pixels can be produced while maintaining the useful features.

\subsection{Spiking Neural Network}

The network used within this paper makes use of both the benefits of convolutional and spiking neural networks. 


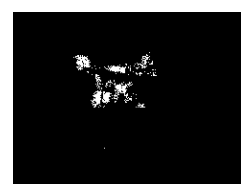

(a)

$200 \mathrm{~ms}$

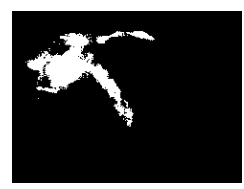

(f)

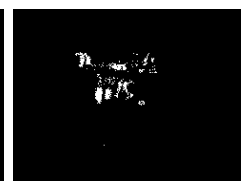

(b)

$100 \mathrm{~ms}$

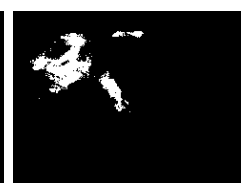

(g)

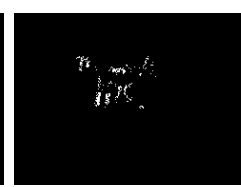

(c)

$50 \mathrm{~ms}$

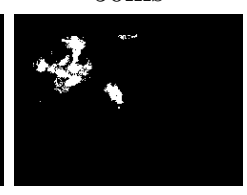

(h)

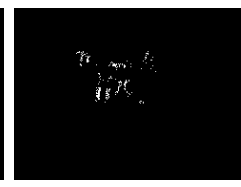

(d)

$30 \mathrm{~ms}$

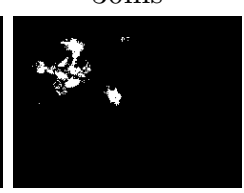

(i)

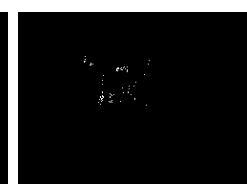

(e)

$10 \mathrm{~ms}$

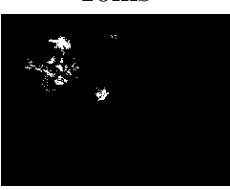

(j)

Fig. 2. DVS filtered events captured in a range of time frames for a low speed (TOP)and high speed scene (BOTTOM)

The CNN brings a local spatial coherence and parameter/weight sharing method, that allows an image to be compressed, such that it can be represented by a respectively smaller number of features versus the number of pixel in the image. Combining these features within progressive layers allows further compression to occur. The SNN allows sparsity to occur through changing of the neuron model, and learning mechanism. The new neuron model converts the floating point values travelling through the network with 1 bit binary spikes. These spikes are far more simplistic in nature, with constant amplitude and duration of individual spikes. Their information is characterised entirely by their emission time (when a neuron fired), and frequency of firing (how often a neuron fires). The neurons have a threshold to reach before passing information forward, but further information can be inferred from the timing of frequency of the neuron firing. In other words, only passing a small amount of important information through the network, but in a timely manner. This can be seen as similar to that of the primate visual system, which has been shown to have spike rates on the order of a few hertz [15]. The change in the neuron model leads to a very important paradigm shift in the network: from looking for content, to looking for context. This reiterates the usefulness of the sparse information transfer that can relate importance into it's time dependency. In that, a few import pieces of context can be used to build content, but no amount of content can give you context. This type of sparse, spike-timebased deep network $[10,12,17]$ is not

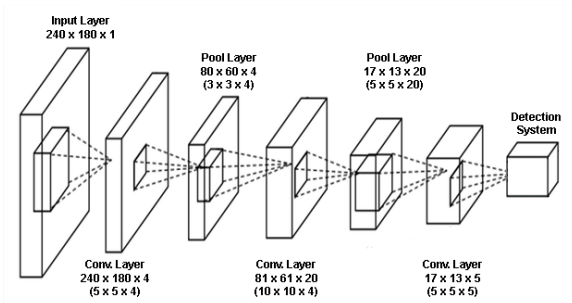

Fig. 3. SNN Architecture 3 Layer Convolution and Pooling as suited for a backpropagation learning mechanism as a CNN. It then makes use of a simplified unsupervised Spike-Time Dependent Plasticity (STDP) rule [2] 
in combination with a winner-takes-all (WTA) approach, to extract hierarchical features in CNN-like architecture. The described network, illustrated in Figure 3, shows the typical three convolution layers with pooling layer in-between. Unlike CNN learning, with STDP each convolution layer has an intra and inter lateral inhibition mechanism $[18,10]$. This helps the network to reduce the information propagated, especially redundant and repeating information, while ensuring that the most salient information is maintained. It operates by only allowing one feature (neuron) in a feature (neuron) map to fire per frame, seen as an intra map competition. This WTA approach then moves onto the inter map inhibition. Only allowing one spike to occur in any given spatial region, typically the size of the convolution kernel, throughout all the maps. When not training the convolution and pooling layers operate in a standard procedure, with the pooling also following the WTA theme with a max pool operation.

\section{Methodology}

Two different methods of capturing data were used in this work. Actual events captured from a DVS and simulated events generated from data captured from a higher resolution DSLR camera. The following describes the set up for the experimental data collection and simulated sensor data creation along with any specifics pertaining to the network and learning mechanism.

\subsection{Dynamic Vision Sensor Data}

The data was captured within a small (basketball court sized) gymnasium, as seen in Figure 4 , in order to be able to control the amount of light in a given scene. Two different sized UAVs (DJI Phantom [without propellers - 290

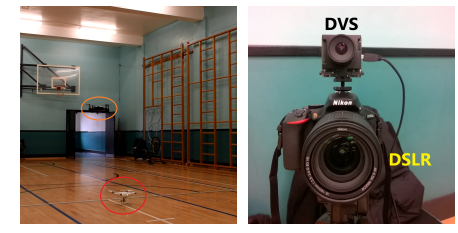

Fig. 4. Indoor Test Set Up x $290 \times 195 \mathrm{~mm}$ ] and DJI Tello [98 x $92.5 \times 41 \mathrm{~mm}]$ ) were used which allowed a wider range of test scenarios to be replicated. The event data was captured using a DVS240 Neuromorphic Vision Sensor with a spatial resolution of 240x180 and asynchronous event output. It was mounted on top of a DSLR Camera, producing a 1920x1080 output at 60 Frames per Second (FPS), both pictured in Figure 4. This was used for ground truth data and use within the simulated data as a means of comparison. The DVS camera is set up to give out a tuple for each spike event, these contain the xy coordinate, the timestamp of when the event occurred, and the polarity of the change in intensity. However, during training and testing of the proposed system the polarity value was ignored, compressing all the spike information into one channel instead of two. The time-stamp data was embedded into each of the frames used for the dataset, this provides a significant advantage over simulated event data [10] as the earlier events are no longer just the highest contrast, but actually, still represent the spatio-temporal domain they were captured in. To further improve this temporal aspect a range of integration time for the dataset frame collection was used, ranging from $10 \mathrm{~ms}$ up to $200 \mathrm{~ms}$ with overlaps in the time windows of $10 \%, 50 \%$ and $90 \%$. This 
wide variety in the frames allows the sensor to capture a diverse range of speed variability within the sensor field of view. This allows the temporal data, usually lost in the snapshot of a frame to be instilled within event capture.

\subsection{Simulated Dynamic Vision Sensor Data}

There are two sources of UAV footage used for the simulated event data: Video recorded from a DSLR camera as explained in the previous section. The other footage is captured from some outside testing using a DSLR(1920x1080 @ 3060fps).

An example of the simulated data is provided in Figure 5, which shows a simulated events frame, along with the pre and post the processing

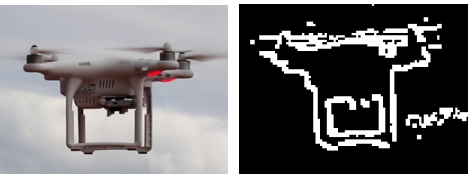

Fig. 5. Simulated UAVs

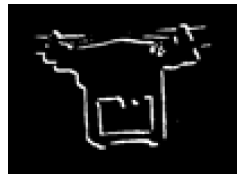

stage when the resolution is down-sampled to $240 \times 180$. The outdoor footage provides a wide range of lighting conditions per frame and a number of different background disturbances to cause noise and clutter in the data (clouds and ground objects). The only issue with simulating the event data is the inher-

Table 1. Table of Network Parameters.

\begin{tabular}{c|ccccc} 
Layer & Conv. 1 & Pool 1 & Conv. 2 & Pool 2 & Conv. 3 \\
\hline Filter Size & 5 & 5 & 10 & 5 & 5 \\
\hline Number of Maps & 4 & 4 & 20 & 20 & 10 \\
\hline Stride & 1 & 5 & 1 & 5 & 1 \\
\hline Propagation Threshold & $\propto$ Input Spikes & 1 & 45 & 1 & 3 \\
\hline Initial Weights & \multicolumn{4}{c}{ Mean of 0.8 with STD 0.08}
\end{tabular}

ent lack of temporal resolution (missing information between each frame that needs to be interpolated). A number of simulators already exist $[3,7,13]$, with PIX2NVS [3] being the event simulator used in this paper. The event sensor simulator takes the frame rate and interpolates the events that would exist between the frames. This is limited to the actual recorded frame rate of the footage, so to further enhance the temporal resolution, some extra post processing is carried out to reduce the number of spikes. Allowing a higher fidelity capture of only the edges of the moving objects.

\subsection{Proposed UAV Detection System}

As mentioned in Section 3.1 the asynchronous data produced by the DVS camera is converted into a frame, embedded with the temporal data (a image where the value of a pixel is the time-stamp of the event occurrence). This frame is then used within a layer-wise learning methodology to extract and build features to allow the network to successfully identify a UAV. A list of the network parameters is shown in Table 1, which also highlights a novel feature of the proposed system, pre-emptive neuron thresholding (PENT). The PENT takes 
the typically reactive neuron thresholding concept [8], but allows it to work in advance of the spikes reaching a neuron. This concept is to overcome the potential of spikes saturating the first layer of the SNN causing a false detection in the system. A typical reactive system would adapt the neuron thresholds if the saturation continued over time, but with the PENT approach, the system is able to act in a timely manner to prevent such saturation from propagating false features through the network. The detection parameters for finding a UAV are embedded within the network itself. As the network enforces a WTA approach to convolution and pooling, the last convolution layer as seen in Figure 3, has a highly sparse input and output. This allows it to act as a detection layer. In this situation, it is able to forgo usage of a fully connected layer [18] or support vector machine [10] as classification isn't required. The network's evaluation will be based upon the number of successful detections and its robustness to a range of highly spiking noisy inputs, replicating low light conditions. The proposed system will use data captured from both the actual DVS and the simulated DVS. The aim is to show how a network can be trained to deliver a higher accuracy from extending training data with simulated DVS data. This data would often be easier to obtain or would already exist, highlighting the ease at which a traditional visual detection system could be converted to an event camera and SNN. With this conversion resulting in a notable reduction in computational, processing and power, promoting its use within an environment where resources are limited.

\section{Testing Results}

This section shows the results of training from three UAV detection networks, using only actual DVS event data, only simulated DVS event data, and our proposed system which utilises both of the previous datasets together. Each of the networks is then tested on a series of actual DVS Event frames, comparing the benefit of additional training data, even if it is simulated data.

DVS Trained Network - During initial testing the network trained on real DVS event data struggled to converge to useful feature within the second layer, due to the sparse feature maps that were learned in the first layer. A set of pre-trained weights representing Gabor features, shown in Figure 6, indicative of that seen in other first layer SNNs $[10,18]$, allowed all the networks to have better building blocks to create more complex features in the second and third layers. Throughout all of the further testing, this

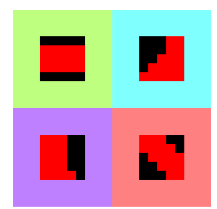

Fig. 6. Synthetic Gabor Features method was used, using the four features presented in Figure 6 as the first layer of each network. Training the network using the events captured from the DVS results in a low resolution feature combination for the second layer. These feature maps resemble low fidelity UAV shapes as seen in Figure 7. It also shows the progression of these shapes into the third layer used 
for detection. This network produced an overall accuracy of $90 \%$ when using PENT (50-54\% with static thresholding depending on the focus of true or false positives). Results of all 3 networks are located in Table 2, showing results of testing against 1,000 UAV images and 1,000 images of empty or noisy scenes (background clutter or a person in the scene).

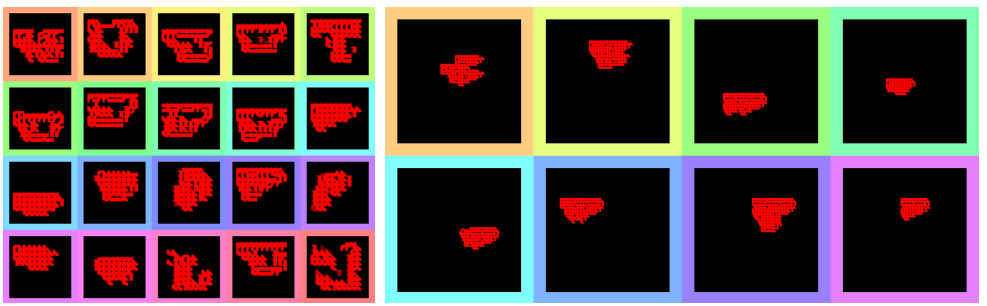

Fig. 7. Second and Third Layer of the Actual DVS Event Trained Network

Table 2. Results data in the confusion matrix for the three trained networks, each being tested with the same 2000 images (1000 with UAV, 1000 without)

\begin{tabular}{|c|c|c|c|c|c|c|c|c|c|}
\hline $\mathrm{N}=2000$ & \multicolumn{8}{|c|}{ UAV Predicted } \\
\hline & & True & False & & True & False & & True & False \\
\hline Actual UAV & True & 850 & 150 & True & 610 & 490 & True & 880 & 120 \\
\hline & False & 50 & 950 & False & 40 & 960 & False & 60 & 940 \\
\hline Overall Accuracy & Actual DVS $=90 \%$ & Simulated DVS $=78 \%$ & Proposed System $=91 \%$ \\
\hline
\end{tabular}

Simulated DVS Trained Network - The network trained using simulated DVS event data was then tested for comparison. These simulated events are originating from a higher resolution image then being scaled to the same resolution as the actual DVS. As the scene is derived from a higher resolution, a higher fidelity feature can occur in the second layer, as seen in Figure 8. These higher fidelity features combine with the low, to create features more representative of a UAV in the third layer. This seemingly qualitative improvement results in a quantitative drop in overall accuracy down to $78 \%$ with full results in Table 2 . The drop in accuracy is a result of the features of the network having to fine a fidelity compared to the actual DVS test set. However, this network did return the best false positive results, suggesting these more complex features were better at discriminating objects in the images without UAVs.

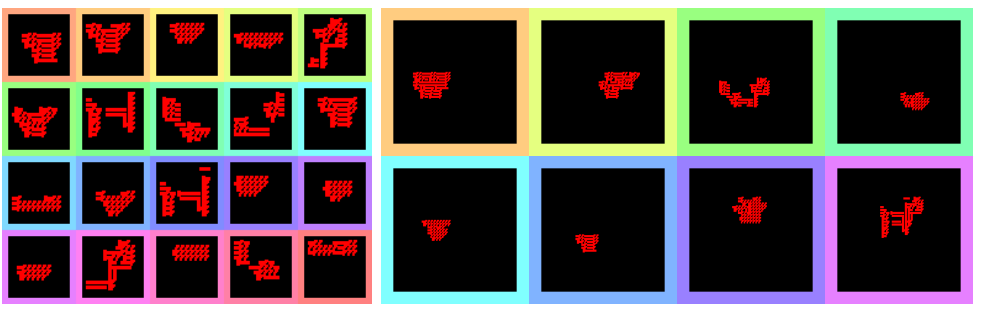

Fig. 8. Second and Third Layer of the Simulated DVS Event Trained Network 
Proposed System Trained Network - The proposed detection system is the third network to be trained, utilising both datasets, real and simulated. At first this network exhibits visually very similar features in the second and third layers as seen in Fig. 9(d) and (h) to that of the simulation data network shown in Figure 8. Figure 9 also demonstrates how the network learns the features seen in these layers, started with the random weight Fig.9(a) and (e), then refining the important features in UAV shaped component parts seen in Fig.9(b),(c),(f) and (g). While the proposed system and previous network trained on simulated data appear to have learned the same feature mapping, the accuracy results show otherwise with an overall accuracy of $91 \%$ exhibiting the highest number of correct detection, results shown in Table 2. To help visualise how these features

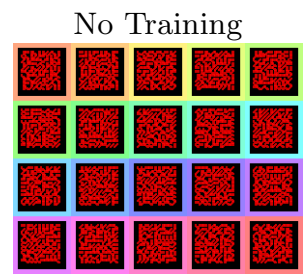

(a)
$1 / 3$ Trained

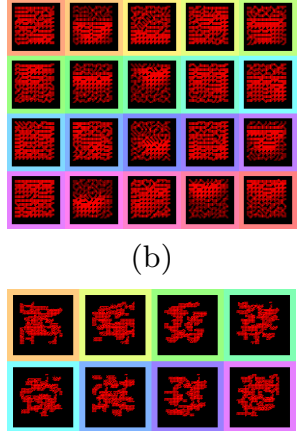

(f)
$2 / 3$ Trained

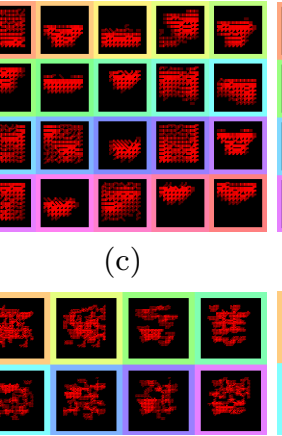

(g)
Fully Trained

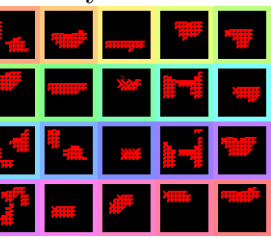

(d)

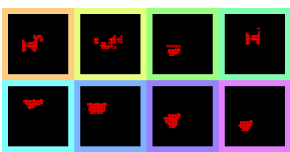

(h)

Fig. 9. Illustration of training in the UAV Detection Network

help to detect the UAV, an example of an event image from both the actual and simulated DVS data is shown in Figure 10, indicating where the pooled mapping of the features map onto the UAV. The image also highlights how an improvement in spatial resolution of the sensor could open up the possibilities of UAV classification system rather than just detection systems. By using the higher fidelity features from the extra spatial resolution, it allows a better realisation on the component part of the UAV allowing more distinct feature to exist.
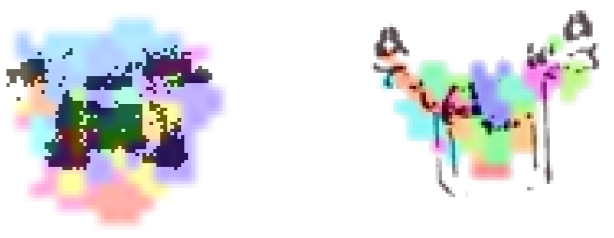

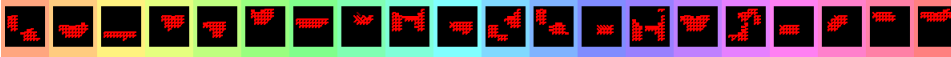

Fig. 10. Feature Mapping and their activations on UAV image 
This demonstrates the main contribution of this paper that simulated DVS events can be a useful training tool for the desired network, when used in conjunction with actual DVS event data, improving upon the network trained only on DVS events. Since traditional video is more regularly available, this can prove an excellent starting point for new ideas and concepts that might not have DVS event footage. This could be ideal in situations where new data is either difficult to generate or obtain.

The proposed system was also able to show robustness to noise with the second contribution of this paper being the introduction of PENT (Pre-Emptive Neuron Thresholding). A visualisation of how noise is handled by the PENT is shown in Figure 11, depicting events captured from a low light scene with a UAV flying, similar to that shown in the low light scene Fig.1 (d). Demonstrating how when PENT is active, only the features of the UAV are captured as shown in Fig.11(b), while when PENT is off, the UAV features are masked by noise seen in Fig.11(f). The reduced propagation of features through the network due to saturation of the fist layer has an impact on all subsequent layers as shown in Fig.11(c),(d),(g) and (h). Testing this feature over a range of noise levels shows that is can maintain an accuracy over $80 \%$ for SNR levels above $1 \mathrm{~dB}$, as seen in Table 3 .

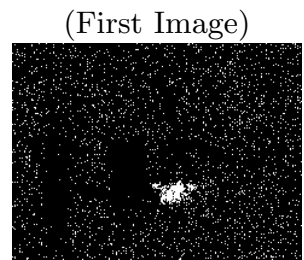

(a)

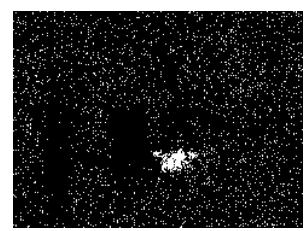

(e)
(First Layer) (Second Layer)
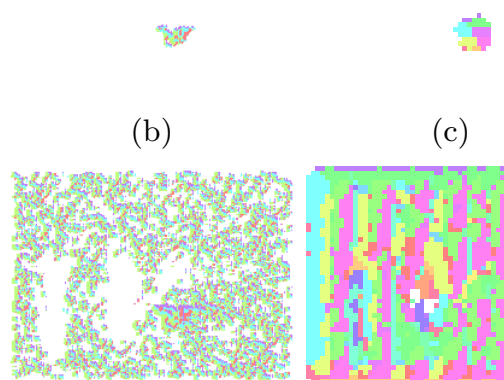

(c)

(f)

(b)

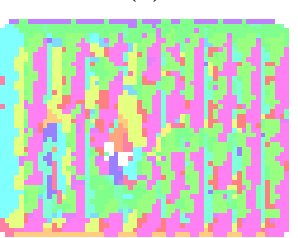

(g)
(Third Layer)

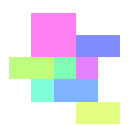

(d)

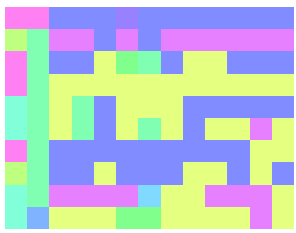

(h)

Fig. 11. Active threshold stops the saturation of layer one features propagation through the network causing false detections.

Table 3. Table of Accuracy with Additive Noise.

\begin{tabular}{c|cccccc} 
SNR Level $(\mathrm{dB})$ & 23 & 15 & 7 & 0.1 & -9 & -15 \\
\hline Accuracy $(\%)$ & 85 & 83 & 82 & 72 & 62 & 46
\end{tabular}

The results from the SNN resemble those from a CNN, thanks partially to the convolution and pooling layers. Furthermore, the system is built upon a 
sparse spiking neuron model which only further sparsifies throughout the network, while in an unsupervised fashion learns distinctive feature to identify a UAV. This sparsity instils the ethos of only transmitting important information, which results in a lower computational throughput, for both runtime and training. On average less than 200 pixels are active per frame that succeeded in detection, a mere $0.5 \%$ of the overall sensor size then moves on for processing. This computation reduction then results in a significant reduction within the SNN compared to a CNN counterpart, with over 270,000 calculation needed for the CNN's convolution layers and only 1,300 required for the SNN on average, over 200 times the difference. A similar reduction in calculation is also seen within the pooling layers thus further reducing the amount of computations needing to be done and resulting in a reduction in power used. This reduction in information transfer allows the system to converge to useful feature quickly. This results in using only 20,000 images each to train the second and third layer (3000 required if you want to train layer one), so 40-43,000 in total.

\section{Conclusion}

Consumer UAVs and micro-UAVs have presented security and defence with a new-age problem. This paper presents a robust detection system for UAVs, that has many of the useful features of other sensors, while fewer of the drawbacks. The overall accuracy of $91 \%$, coupled with an enhanced resilience to noise due to PENT, make the proposed system a feasible alternative for the future. From utilising the sparse nature of the SNN, this accuracy comes with the benefit of also providing a far lower computation load than a traditional CNNs, this being a result of not having to pass information from every neuron in the layer, but only those who pass the threshold. The SNN also pairs nicely with the asynchronous event driven nature of the DVS. With its output also representing a sparse version of the traditional frame based camera. The system to that effect then delivers high accuracy, while being the sparse version of the traditional system. This sparsity can deliver many benefits with reductions in computational processing leading to a reduction in overall size, weight, power and cost, therefore improving overall application system viability.

\section{References}

1. BBC News: Gatwick Airport: Drones ground flights - BBC News, https://www.bbc.co.uk/news/uk-england-sussex-46623754

2. Bi, G.q., Poo, M.m.: Synaptic Modifications in Cultured Hippocampal Neurons: Dependence on Spike Timing, Synaptic Strength, and Postsynaptic Cell Type. Journal of Neuroscience 18(24), 10464-10472 (dec 1998). https://doi.org/10.1523/JNEUROSCI.18-24-10464.1998

3. Bi, Y., Andreopoulos, Y.: PIX2NVS: Parameterized conversion of pixel-domain video frames to neuromorphic vision streams. In: 2017 IEEE International Conference on Image Processing (ICIP). pp. 1990-1994. IEEE (sep 2017). https://doi.org/10.1109/ICIP.2017.8296630 
4. Brandli, C., Berner, R., Minhao Yang, Shih-Chii Liu, Delbruck, T.: A 240 x $180130 \mathrm{~dB} 3 \mu \mathrm{s}$ Latency Global Shutter Spatiotemporal Vision Sensor. IEEE Journal of Solid-State Circuits 49(10), 2333-2341 (oct 2014). https://doi.org/10.1109/JSSC.2014.2342715

5. Civil Aviation Authority: Airprox involving UAS Drones - UK Airprox Board (2019), https://www.airproxboard.org.uk/Reports-andanalysis/Statistics/Airprox-involving-UAS-Drones

6. G4S: Drones: Threat from Above. Tech. rep. (2017), www.g4s.us

7. Garcia, G.P., Camilleri, P., Qian Liu, Furber, S.: pyDVS: An extensible, realtime Dynamic Vision Sensor emulator using off-the-shelf hardware. In: 2016 IEEE Symposium Series on Computational Intelligence (SSCI). pp. 1-7. IEEE (dec 2016). https://doi.org/10.1109/SSCI.2016.7850249

8. Huang, C., Resnik, A., Celikel, T., Englitz, B.: Adaptive Spike Threshold Enables Robust and Temporally Precise Neuronal Encoding. PLOS Computational Biology 12(6), e1004984 (jun 2016). https://doi.org/10.1371/journal.pcbi.1004984, https://dx.plos.org/10.1371/journal.pcbi.1004984

9. Huang, J., Guo, M., Chen, S.: A dynamic vision sensor with direct logarithmic output and full-frame picture-on-demand. In: 2017 IEEE International Symposium on Circuits and Systems (ISCAS). pp. 1-4. IEEE (may 2017). https://doi.org/10.1109/ISCAS.2017.8050546

10. Kheradpisheh, S.R., Ganjtabesh, M., Thorpe, S.J., Masquelier, T.: STDPbased spiking deep convolutional neural networks for object recognition. Neural Networks 99, 56-67 (mar 2018). https://doi.org/10.1016/J.NEUNET.2017.12.005, https://www.sciencedirect.com/science/article/pii/S0893608017302903?via\%3Dihub

11. Mueggler, E., Rebecq, H., Gallego, G., Delbruck, T., Scaramuzza, D.: The EventCamera Dataset and Simulator: Event-based Data for Pose Estimation, Visual Odometry, and SLAM. Tech. rep. (2016), http://rpg.ifi.uzh.ch/davis_data.html

12. Panda, P., Srinivasan, G., Roy, K.: Convolutional Spike Timing Dependent Plasticity based Feature Learning in Spiking Neural Networks. Tech. rep. (2017), https://arxiv.org/pdf/1703.03854.pdf

13. Rebecq, H., Gehrig, D., Scaramuzza, D.: ESIM: an Open Event Camera Simulator. Tech. rep. (2018), https://www.blender.org/

14. Shakhatreh, H., Sawalmeh, A., Al-Fuqaha, A., Dou, Z., Almaita, E., Khalil, I., Othman, N.S., Khreishah, A., Guizani, M.: Unmanned Aerial Vehicles: A Survey on Civil Applications and Key Research Challenges (2018)

15. Shoham, S., O'Connor, D.H., Segev, R.: How silent is the brain: is there a "dark matter" problem in neuroscience? Journal of Comparative Physiology A 192(8), 777-784 (aug 2006). https://doi.org/10.1007/s00359-006-0117-6

16. Son, B.e.a.: 4.1 A $640 \times 480$ dynamic vision sensor with a $9 \mu \mathrm{m}$ pixel and 300Meps address-event representation. In: 2017 IEEE International Solid-State Circuits Conference (ISSCC). pp. 66-67. IEEE (feb 2017). https://doi.org/10.1109/ISSCC.2017.7870263

17. Tavanaei, A., Maida, A.S.: BIO-INSPIRED SPIKING CONVOLUTIONAL NEURAL NETWORK USING LAYER-WISE SPARSE CODING AND STDP LEARNING. Tech. rep. (2017), https://arxiv.org/pdf/1611.03000.pdf

18. Thiele, J.C., Bichler, O., Dupret, A.: Event-Based, Timescale Invariant Unsupervised Online Deep Learning With STDP. Frontiers in Computational Neuroscience 12, 46 (jun 2018). https://doi.org/10.3389/fncom.2018.00046

19. UK, D.f.T.: Taking Flight: The Future of Drones in the UK Government Response (2019), https://assets.publishing.service.gov.uk/government/uploads/future-ofdrones-in-uk-consultation-response-web.pdf 